\title{
Composição Eficiente de Encadeamento de Funções de Serviços
}

\author{
Igor Ferrazza Capeletti ${ }^{1}$, Fabio Diniz Rossi ${ }^{2}$, Marcelo Caggiani Luizelli ${ }^{1}$ \\ ${ }^{1}$ Universidade Federal do Pampa (UNIPAMPA) \\ ${ }^{2}$ Instituto Federal Farroupilha (IFFAR)
}

A implantação dos serviços de rede por partes dos provedores (ISP Internet Service Providers) é focada em atender padrões de tráfego existentes [Gil Herrera and Botero 2016]. No entanto, com a flexibilidade e a agilidade vislumbradas pelos paradigmas NFV (Network Function Virtualization) e SDN (Softwaredefined Networks), o provisionamento de serviços de rede se torna possível a uma granularidade muito menor. Por exemplo, é concebível imaginar (num futuro próximo) implantações de serviço de rede adaptadas às necessidades dos clientes ou mesmo por conexões individuais (ao invés de serem adaptadas para tráfego de rede genéricos).

No entanto, até mesmo os recentes paradigmas NFV e SDN ainda enfrentam desafios técnicos para atingir o nível necessário de desempenho, agilidade e custobenefício. Nesse contexto, um dos desafios mais importantes é a sobrecarga de virtualização [Luizelli et al. 2017b]. Por um lado, NFV ainda depende do uso de mecanismos de virtualização tradicionais e monolíticos para implementar funções de rede virtualizadas (VNF - Virtual Network Functions). No caso de um ambiente dinâmico e em larga escala, essas técnicas tradicionais de virtualização não são adequadas para garantir os níveis necessários de desempenho e agilidade. Apesar de seus benefícios bem conhecidos (por exemplo, isolamento de recursos), ainda há muitas desvantagens desse nível de virtualização aplicado ao NFV - por exemplo, alto tempo de inicialização de VNFs (na ordem de segundos) e grande quantidade de memória utilizada.

Por outro lado, o encadeamento dinâmico e flexível fornecido por ambientes SDN/NFV dependem fortemente das camadas de encaminhamento em software para permitir a comunicação de um conjunto de VNFs. Embora implementações de switches em software (por exemplo, Open vSwitch [Pfaff et al. 2015]) não sejam restritas diretamente aos recurso físicos (por exemplo, memória física TCAM), existe degradação de desempenho quanto a capacidade de encaminhamento - a qual depende da arquitetura física subjacente [Luizelli et al. 2018]. Exemplos incluem o uso excessivo de tabelas de encaminhamento e classificação de tráfego em uma granularidade menor. O primeiro (uso excessivo de tabelas de encaminhamento) leva a degradação do desempenho, pois nesse caso há constantemente acesso a subsistemas de memória com custos maiores (por exemplo, memória DRAM) em vez de mecanismos de armazenamento em cache (por exemplo, L1 e L2). No segundo, a classificação de tráfego precisa de mais cabeçalhos para serem extraídos/processados, o que aumenta diretamente o tempo de processamento do pacote no pipeline do plano de dados.

Avanços recentes em tecnologias de aceleração de pacotes (por exemplo, Intel DPDK $^{1}$ ) e o desenvolvimento de máquinas virtuais minimalistas (por exemplo, Unikernels) estão impulsionando os esforços para superar os desafios gerais de virtualização aplicadas ao contexto de NFV. No entanto, a fim de materializar serviços ágeis baseados em NFV com baixa granularidade (por exemplo, em nível de fluxo), o meca-

\footnotetext{
${ }^{1}$ http: / / dpdk.org/
} 
nismo de escalonamento na camada do orquestrador NFV deve ser extremamente escalável e eficiente. A literatura recente tem se concentrado na implantação atômica de cadeias de serviços [Wenrui et al. 2017, Luizelli et al. 2017a] - com pouco ou nenhum compartilhamento/sobreposição com serviços de rede já implantados. Na prática, os ISPs necessitam de mecanismos mais ágeis para programar fluxos de rede para serviços de rede já implantados (por meio de redirecionamento de tráfego) ou implantar novas cadeias de serviços quando necessário para lidar com as demandas de fluxo de rede.

Para prover um mecanismo de escalonamento de serviços NFV eficiente e ágil em nível de fluxo, argumenta-se que é necessário utilizar os avanços recentes na virtualização de rede e a composição incremental da cadeia de serviços [Beck and Botero 2015, Beck and Botero 2017]. O primeiro permite que o orquestrador NFV gerencie o ciclo de vida de VNFs na ordem de milissegundos (ou mesmo micro). Por sua vez, o segundo permite que o tempo de provisionamento seja substancialmente reduzido, compartilhando sempre que possível as VNFs já provisionada e regras de encaminhamento. Neste trabalho, dá-se o primeiro passo na direção de algoritmos eficientes para a composição eficaz de serviços de rede. Propõem-se uma heurística gulosa capaz de agregar (ou realizar o merge) de encadeamentos de serviços de rede de maneira online. A heurística objetva minimizar o tempo de provisionamento de requisições de encadeamentos de VNFs, minimizando o consumo geral de recursos utilizados da infraestrutura física. $\mathrm{O}$ algoritmo é comparado com trabalhos existentes da literatura (por exemplo, [Luizelli et al. 2017a]) que realizam o provisionamento atômico de encadeamentos de VNFs.

\section{Referências}

Beck, M. T. and Botero, J. F. (2015). Coordinated allocation of service function chains. In 2015 IEEE Global Communications Conference (GLOBECOM), pages 1-6.

Beck, M. T. and Botero, J. F. (2017). Scalable and coordinated allocation of service function chains. Computer Communications, 102:78 - 88 .

Gil Herrera, J. and Botero, J. F. (2016). Resource allocation in nfv: A comprehensive survey. IEEE Transactions on Network and Service Management, 13(3):518-532.

Luizelli, M. C., da Costa Cordeiro, W. L., Buriol, L. S., and Gaspary, L. P. (2017a). A fixand-optimize approach for efficient and large scale virtual network function placement and chaining. Computer Communications, 102:67 - 77.

Luizelli, M. C., Raz, D., and Sa'ar, Y. (2018). Optimizing nfv chain deployment through minimizing the cost of virtual switching. In IEEE INFOCOM 2018 - IEEE Conference on Computer Communications, pages 2150-2158.

Luizelli, M. C., Raz, D., Sa'ar, Y., and Yallouz, J. (2017b). The actual cost of software switching for nfv chaining. In 2017 IFIP/IEEE Symposium on Integrated Network and Service Management (IM), pages 335-343.

Pfaff, B., Pettit, J., Koponen, T., Jackson, E., Zhou, A., Rajahalme, J., Gross, J., Wang, A., Stringer, J., Shelar, P., Amidon, K., and Casado, M. (2015). The design and implementation of open vswitch. In 12th USENIX Symposium on Networked Systems Design and Implementation (NSDI 15), pages 117-130, Oakland, CA. USENIX Association.

Wenrui, M., Sandoval, O., Beltran, J., Pan, D., and Pissinou, N. (2017). Traffic aware placement of interdependent $\mathrm{nfv}$ middleboxes. In IEEE International Conference on Computer Communications (INFOCOM 2017), Atlanta, USA. 